\title{
Laser-Desorbed Large Molecules in a Supersonic Jet
}

\author{
H. v. Weyssenhoff*, H. L. Selzle, and E. W. Schlag ** \\ Institut für Physikalische und Theoretische Chemie der Technischen Universität München, \\ Garching
}

\author{
Z. Naturforsch. 40a, 674-676 (1985); received April 29, 1985
}

\begin{abstract}
A new technique of desorption of non volatile thermally unstable molecules into a supersonic jet has been developped. With this method isolated large molecules are now available for mass spectrometry as well as for spectroscopic studies at temperatures of a few Kelvin, thus avoiding spectral congestion. It could be demonstrated that molecules like tryptophane and retinal could be desorbed with an IR laser pulse without decomposition and be ionized with a second UV laser to form bare molecule ions without fragmentation.
\end{abstract}

Spectra of large molecules in solution generally display only a broad band response with little detailed information, in contrast to gas phase spectra which give detailed structural and dynamic information of very many lines. Hence, by vaporizing large molecules one expects an enormous increase in the structural information obtainable. Experiments with some thermally stable systems have confirmed this. Unfortunately many, if not most large molecules are thermally unstable so that special techniques must be found for volatization if extensive fragmentation is to be avoided. Once volatalized the molecules can remain in the gas phase if they are diluted in an inert medium. This medium can be isentropically expanded in a supersonic beam which then yields temperatures in the $1 \mathrm{~K}$ range. This further simplifies the spectra of most large molecules which are otherwise invariably susceptible to hot band congestion.

A suitable method for injecting large thermally unstable non-volatile molecules into a supersonic beam of a noble gas therefore would make such molecules accessible to electronic spectroscopy under collisionfree conditions in a state of frozen internal modes of motion. Here we employ a two laser method, one to vaporize and one to ionize the sample. This two step technique has certain advantages over a one step ionization method.

In recent years a number of different techniques for mass spectrometry [1] have been developed to

\footnotetext{
* Permanent address: Institut für Physikalische Chemie der Universität Hannover.

** Reprint requests to Prof. Dr. E. W. Schlag, Institut für Physikalische und Theoretische Chemie der TU München, Lichtenbergstraße 4, D-8046 Garching.
}

desorb non-volatile substances directly from a solid sample by irradiation with photons, electrons or fast ionic or neutral particles. The interaction may probably be most closely described as ultrafast local heating at the surface of the sample which results in desorption of neutral and charged species. In most cases only a small fraction of the desorbed particles is ionized and subjected to mass spectrometry.

Here we report preliminary mass spectra [2] of some thermally unstable non-volatile compounds which were injected by IR-LD into a supersonic jet and ionized by MPI. In particular, IR Laser-induced photodesorption (LD) [3] can yield exclusively neutral molecules [4] without significant fragmentation when moderate laser fluences are used. The test substances were tryptophane ( $\alpha$-amino- $\beta$-indoylpropionic acid) and retinal (Vitamine A aldehyde) as well as anthracene as a reference with well known spectroscopic data.

A pulsed supersonic argon jet was generated by a modified fuel injection valve (Bosch Mod. 280150152). The valve throat itself constituted an annular nozzle of ca. $100 \mu \mathrm{m}$ width which was capable of forming a cold jet without an additional pinhole nozzle. The laser desorption took place in a mixing chamber mounted immediately in front of the valve throat as shown in Figure 1. The chamber consisted of a cylindrical block of stainless steel with an axial bore of $2.5 \mathrm{~mm}$ diameter through which the expanding carrier gas pulse passes. Perpendicular to this bore a channel of $2.5 \mathrm{~mm}$ was drilled; thus the sample could be irradiated inside the mixing chamber with a focused laser pulse. The samples consisted of bulk material pressed to a pill. The expanded supersonic jet was directly introduced into the

0340-4811 / 85 / 0700-0674 \$01.30/0. - Please order a reprint rather than making your own copy. 


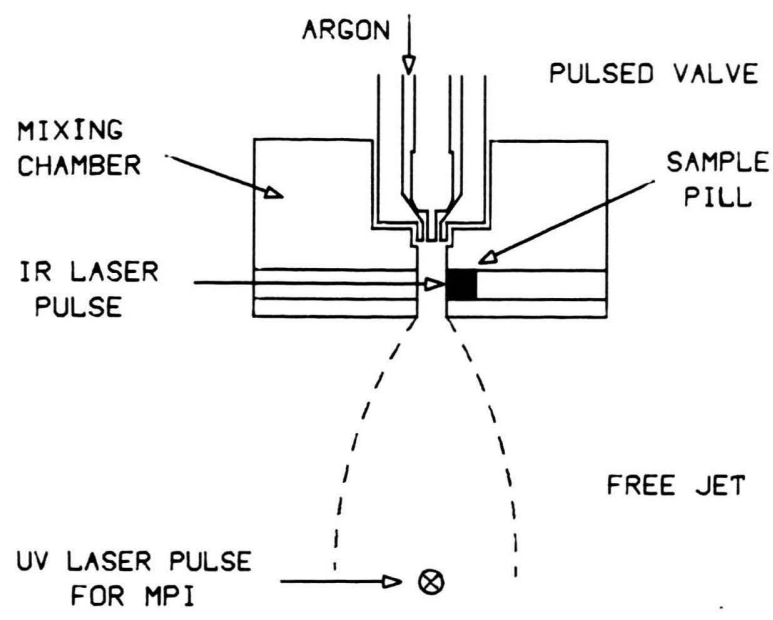

Fig. 1. Mixing chamber for laser desorption into a supersonic jet.

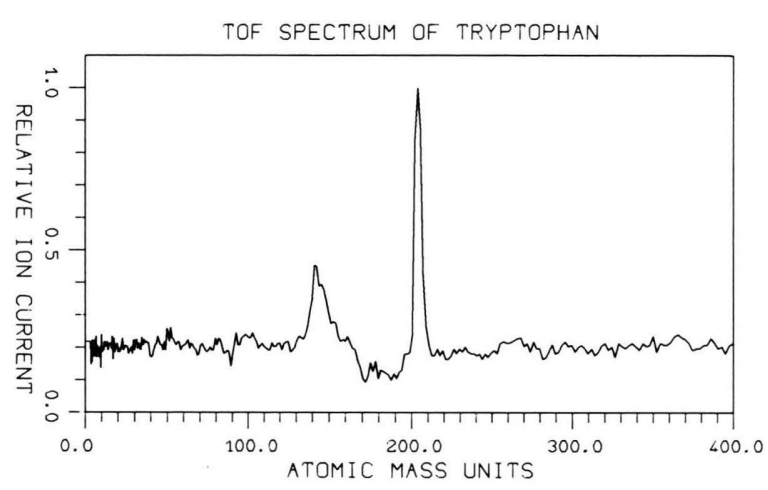

Fig. 2. Time-of-Flight mass spectrum of laser desorbed tryptophan (mass 204). Ionisation laser at $\lambda=266 \mathrm{~nm}$.

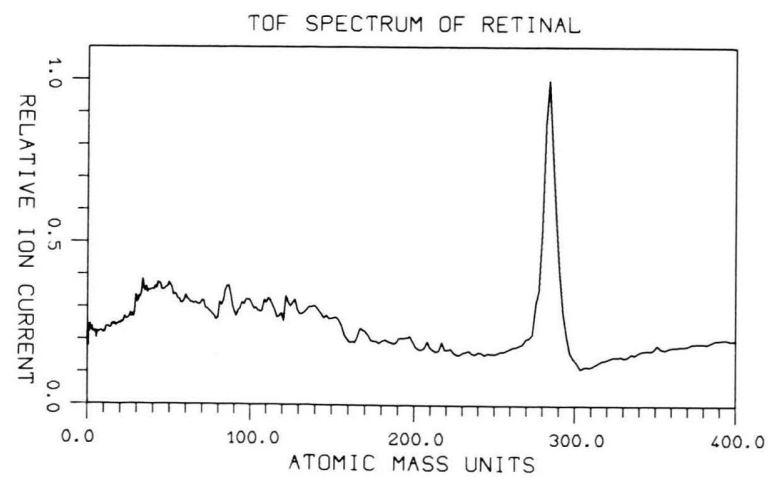

Fig. 3. Time-of-Flight mass spectrum of laser desorbed retinal (mass 284). Ionization laser at $\lambda=355 \mathrm{~nm}$. drawout region of a time-of-flight mass spectrometer in a threegrid configuration (Wiley-McLaren) [5]. The drift tube of the mass spectrometer was pumped differentially. A secondary electron multiplier was used as a detector. The mass spectra were recorded on a Tektronix 7912 transient digitizer. For LD a pulsed $\mathrm{CO}_{2}$-Laser (Lumonics) capable of delivering pulses with a rectangular cross section of $2.3 \times 2.5 \mathrm{~cm}^{2}$, up to $0.3 \mathrm{~J} / \mathrm{cm}^{2}$ in $1 \mu \mathrm{s}$ was used. The beam was focussed by a gold covered concave mirror $(f=28 \mathrm{~cm})$ and directed to the sample through a barium fluoride window. The intensity of the laser pulse could be controlled by two variable iris apertures placed in the beam prior to focussing. MPI was produced by a Quanta Ray pulsed NdYAG and dye laser system using frequency doubling. The laser pulse was focussed to the crossing point of the jet with the ion optical axis of the TOFMS by means of a quartz lens $(f=50 \mathrm{~cm})$. Synchronisation of the pulsed valve, the two lasers and the transient digitizer was accomplished by separately adjustable delay generators.

In a preliminary experiment a LIF excitation spectrum of laser-desorbed anthracene was taken by tuning the dye laser across the $0-0$ band. The width of the resonance band was approx. $0.1 \mathrm{~nm}$ corresponding to an internal temperature of a few Kelvin. This proved that cooling is fully effective even for molecules injected by LD into the expanding supersonic jet.

Non-resonant MPI mass spectra of tryptophan and retinal were taken under the following experimental conditions: argon stagnation pressure: 0.4 bar; power density of the $\mathrm{CO}_{2}$-laser: ca. $1.2 \mathrm{MW} / \mathrm{cm}^{2}$; delay from valve opening time to the $\mathrm{CO}_{2}$-laser pulse: $880 \mu \mathrm{s}$; and a delay further of the ionizing laser pulses: $350 \mu$ sd. The recorded spectra were averaged over 10 shots. The mass spectrum shown in Fig. 2 was taken with the ionizing laser wavelength at $266 \mathrm{~nm}$ (4th harmonic of the Nd-YAG laser) at a power level just high enough to cause ionization. Similarly, the mass spectrum of retinal in Fig. 3 was obtained with a laser wavelength of $355 \mathrm{~nm}$. When the power density was increased considerable ion fragmentation was observed in both cases. Both spectra show essentially the parent ion peak with no indication of significant fragmentation due to the IR laser desorption process. The small additional features in the mass spectra are due to an impurity background which was also present in the 
absence of the IR laser pulse. These results clearly demonstrate the usefulness of IR-Laser-induced desorption for seeding a supersonic jet with molecules of thermally unstable non-volatile compounds.

Recently, Rizzo, Park, and Levy [6] reported the production of a jet seeded with tryptophane employing, however, the thermospray technique [7]. Using a solution in methanol they obtained a mass spectrum from MPI in the skimmed jet showing the parent ion mass but also an almost equal breakdown peak. The spectrum also showed two peaks due to tryptophane-methanol complexes. Since thermosprays can also produce ions [7] it seems likely that

[1] G. D. Davis, Acc. Chem. Res. 12, 359 (1979); R. D. Mcfarlane, Acc. Chem. Res. 15, 268 (1982), and references cited therein.

[2] W. E. Henke, H. V. Weyssenhoff, H. L. Selzle, and E.W. Schlag, Paper M 138 presented at the meeting of the Deutsche Physikalische Gesellschaft, Regensburg, 1983 and published in Verhandl. Deutsch. Phys. Ges. 3/1983, p. 341, Patent Ne. P 4392 PCT EP (pending).

[3] a) Review articles: F. Hillekamp, in: Laser Induced Ion Formation from Organic Solids, ed. A. Benninghoven, the fragments originate in the thermospray rather than the MIP process. Peaks due to fragment ions were not observed in our experiments (our secondary peaks are always present and due to a residual contamination of the system). While the thermospray technique offers an interesting alternative to the LD-method described here further work is needed to allow a full evaluation.

One of the authors (H.v.W.) gratefully acknowledges financial support by the Deutsche Forschungsgemeinschaft during his sabbatical term at the TU München.
Springer Ser. in Chem. Phys. 25, 190 (1983); R. J. Cotter, Anal. Chem. 56, 488 A, (1984); b) J. C. Tabet and R. J. Cotter, Anal, Chem. 56, 1662 (1984).

[4] C. H. Becker and K. T. Gillen, Anal. Chem. 56, 1671 (1984).

[5] W. C. Wiley and I. H. Mclaren, Rev. Sci. Instrum. 26, 1150 (1955).

[6] T. R. Rizzo, Y. D. Park, and D. H. Levy, J. Amer. Chem. Soc. 107, 277 (1985).

[7] C. R. Blakley and M. L. Vestal, Anal. Chem. 55, 750 (1983), and references cited therein. 